\title{
Null Cartan curve variations in 3D semi-Riemannian manifold
}

\author{
Zehra Özdemir \\ Department of Mathematics, Faculty of Science and Arts, Amasya University, Amasya, Turkey
}

\begin{abstract}
The aim of this study is to investigate the variations of the Bishop frame curvatures for null Cartan curves in semi-Riemannian manifolds. The Killing equations in terms of the variations of the Bishop curvatures along the null Cartan curve is especially derived. Killing equations are used to interpret the movement of the charged particles in a magnetic field. The charged particle motion along a null Cartan curve through the Killing equations is examined as an application in the study. It is found that the charged particle traces a trajectory in the form of the null cubic during its movement in the Killing magnetic vector field. According to the results obtained, an example of the null Cartan magnetic trajectories is presented.
\end{abstract}

Mathematics Subject Classification (2020). 14H45, 57R25, 51B20

Keywords. variation, killing vector field, Bishop frame, Cartan frame, null Cartan curve

\section{Introduction}

There are three types of curves: spacelike, timelike, and null (lightlike) in semiRiemannian manifolds. The spacelike and timelike curves may have a similar characterization whereas the null curve is different among these curves because of the metrical properties. While the induced metric on the spacelike and timelike curves are non-degenerate, the induced metric on null curves is degenerate. The relativistic particle models are expressed by actions derived on a null curve. In [7-9], the authors investigate these actions in Minkowski space. Moreover, they solved the variational problem of a null curve and they found that the solution was a null elastic curve resulting via the rigid motion in the rotational Killing vector field direction. Furthermore, the real classical string in Minkowski space was associated with the null curves (see [11]). The string equations simplify the wave equations. The solution of the 2-dimensional wave equation have presented that strings are equal to a single null curve or pairs of null curves (see also $[11-13,20,21]$ ). In addition, since Killing vector fields are divergence-free, they define a magnetic vector field called a Killing magnetic vector field. If a charged particle enters a magnetic vector field, then the velocity field of the curve exposes to the magnetic vector field. Thus, the trajectory of the charged particle changes. This new trajectory of the particle is called a magnetic trajectory. The charged particle motion using the variational problem has been

Email address: zehra.ozdemir@amasya.edu.tr

Received: 23.05.2019; Accepted: 16.06.2020 
examined in Euclidean 3-space and Lorentzian 3-space by Barros [1,2]. All these studies have resulted in the analysis to be conducted on variational problems on null curves.

The study is composed of six sections as follows: In Section 2, some of the necessary concepts are reviewed. In Section 3, the variational formulas for the Bishop curvatures of the null Cartan curve is derived. This section also involves a discussion about the relationship between the variational formulas of the Bishop curvatures and the Killing equations along a null Cartan curve in semi-Riemannian space forms. In Section 4, the results are used to examine the charged particle trajectories in the Killing magnetic vector field. Moreover, an example of null Cartan magnetic trajectory is given to illustrate the theoretical results. In Section 5, the physical meaning of the results are summarized. The final section includes conclusions and discussion.

\section{Preliminaries}

A semi-Riemannian manifold $(M, \wp)$ is defined with a non-degenerate metric tensor $\wp$ with signature $(-,+,+)$ given by

$$
\wp(\omega, \varpi)=-\omega_{1} \varpi_{1}+\omega_{2} \varpi_{2}+\omega_{3} \varpi_{3},
$$

for all $\omega=\left(\omega_{1}, \omega_{2}, \omega_{3}\right), \varpi=\left(\varpi_{1}, \varpi_{2}, \varpi_{3}\right) \in \chi(M)$.

The sectional curvature of a non-degenerate plane spanned by $\{\omega, \varpi\}$ is defined by

$$
K(\omega, \varpi)=\frac{\wp(\Re(\omega, \varpi) \omega, \varpi)}{\wp(\omega, \omega) \wp(\varpi, \varpi)-\wp(\omega, \varpi)^{2}},
$$

where $\Re$ is semi-Riemannian curvature tensor given by

$$
\Re\left(U_{1}, U_{2}\right) U_{3}=-\nabla_{U_{1}} \nabla_{U_{2}} U_{3}+\nabla_{U_{2}} \nabla_{U_{1}} U_{3}+\nabla_{\left[U_{1}, U_{2}\right]} U_{3},
$$

$U_{i} \in \chi(M), i=1,2,3$. Semi-Riemannian manifold with constant sectional curvature is called semi-Riemannian space form. Then, the curvature tensor $\Re$ is given following formula in this manifold:

$$
\Re\left(U_{1}, U_{2}\right) U_{3}=C\left\{\wp\left(U_{3}, U_{1}\right) U_{2}-\wp\left(U_{3}, U_{2}\right) U_{1}\right\},
$$

where $C$ is the constant sectional curvature.

A non-zero vector $\omega \in \chi(M)$ is called space-like if $\wp(\omega, \omega)>0$, time-like if $\wp(\omega, \omega)<0$ and null if $\wp(\omega, \omega)=0, \omega \neq 0$. Any two vectors $\omega, \varpi \in \chi(M)$ are called orthogonal if $\wp(\omega, \varpi)=0$. Two null vectors are orthogonal on the condition that they are linearly dependent.

Let $\beta$ be a smooth curve in $(M, \wp)$ with the immersion $\imath: \beta \rightarrow M$. Let $U$ be a coordinate neighborhood on $M$ and $w$ be the corresponding local parameter. Then $\beta$ is expressed by the following representation

$$
\beta: I \rightarrow M ; w \rightarrow \beta(w),
$$

where $I$ is an open interval of $\mathbb{R}$.

The curve $\beta$ is called a null (isotropic or light-like) curve on the condition that the velocity vector field along the curve $\beta$ is a null vector. Namely, it is as follows;

$$
g\left(\frac{d \beta}{d w}, \frac{d \beta}{d w}\right)=0 .
$$

A null curve $\beta$ is said to be a null Cartan curve, if it satisfies

$$
s(w)=\int_{0}^{w} g\left(\beta^{\prime \prime}(w), \beta^{\prime \prime}(w)\right) d w,
$$


where $s$ is called pseudo-arc parameter. The Cartan frame $\{t, n, b\}$ of a non-geodesic null Cartan curve $\beta$ satisfies the following equations;

$$
\begin{aligned}
t^{\prime} & =\kappa n, \\
n^{\prime} & =-\kappa t+\tau b, \\
b^{\prime} & =-\tau b,
\end{aligned}
$$

where the first Cartan curvature $\kappa(s)=1$ and the torsion $\tau(s)$ is an arbitrary function according to the pseudo-arc parameter $s$. The null Cartan curves are called null Cartan cubics on the condition that $\tau(s)=0$. The null Cartan frame vectors satisfy the following relation,

$$
\begin{aligned}
\wp(t, t) & =\wp(b, b)=0, \wp(n, n)=1, \\
\wp(t, n) & =\wp(n, b)=0, \wp(t, b)=-1,
\end{aligned}
$$

and

$$
t \times n=-t, n \times b=-b, b \times t=n,
$$

[6]. The Frenet frame is constructed for differentiable non-degenerate curves for 3-times continuously. However, the second derivative of the curve may be zero at any point of the curve. In this case, we can define an alternative frame called Bishop frame. The Bishop frame $t, n_{1}, n_{2}$ of a regular curve in 3-dimensional Euclidean space is introduced by Bishop, [3]. It consists of a tangent vector field $t$ and two relatively parallel vector field $n_{1}$ and $n_{2}$, (see [3]). In Minkowski 3-space the Bishop frame for non-null curves is obtained in [18]. For null and pseudo null curve, the Bishop frame defined as in the following theorem.

Theorem 2.1 ([10]). Let $\beta$ be a null Cartan curve in $(M, \wp)$ parameterized by pseudo-arc $s$ with the Cartan curvatures $\kappa(s)=1$ and the torsion $\tau(s)$. Then, the relation with the Bishop frame $\left\{t_{1}, n_{1}, n_{2}\right\}$ and the Cartan frame $\{t, n, b\}$ of the curve $\beta$ are given as follows:

$$
\left[\begin{array}{c}
t_{1} \\
n_{1} \\
n_{2}
\end{array}\right]=\left[\begin{array}{ccc}
1 & 0 & 0 \\
-k_{2} & 1 & 0 \\
\frac{k_{2}^{2}}{2} & -k_{2} & 1
\end{array}\right]\left[\begin{array}{c}
t \\
n \\
b
\end{array}\right]
$$

and the Bishop frame equations for null Cartan curve $\beta$ defined as follows;

$$
\left[\begin{array}{c}
t_{1}^{\prime} \\
n_{1}^{\prime} \\
n_{2}^{\prime}
\end{array}\right]=\left[\begin{array}{ccc}
k_{2} & k_{1} & 0 \\
0 & 0 & k_{1} \\
0 & 0 & -k_{2}
\end{array}\right]\left[\begin{array}{c}
t_{1} \\
n_{1} \\
n_{2}
\end{array}\right],
$$

where the first Bishop curvature $k_{1}(s)=1$ and the second Bishop curvature $k_{2}(s)$ satisfies the following differential equation;

$$
k_{2}^{\prime}(s)=-\frac{1}{2} k_{2}^{2}(s)-\tau(s) .
$$

The Bishop frame $\left\{t_{1}, n_{1}, n_{2}\right\}$ satisfies the following conditions;

$$
\begin{aligned}
& \wp\left(t_{1}, t_{1}\right)=\wp\left(n_{2}, n_{2}\right)=0, \wp\left(n_{1}, n_{1}\right)=1, \\
& \wp\left(t_{1}, n_{1}\right)=\wp\left(n_{1}, n_{2}\right)=0, \wp\left(t_{1}, n_{2}\right)=-1,
\end{aligned}
$$

Moreover, the cross product of the Bishop frame vectors satisfies the following condition;

$$
t_{1} \times n_{1}=-t_{1}, n_{1} \times n_{2}=-n_{2}, n_{2} \times t_{1}=n_{1},
$$

[10]. 


\section{Null Bishop variations in semi-Riemannian manifold $(M, \wp)$}

Lemma 3.1. Let $\beta: I \subset \mathbb{R} \rightarrow M$ be a null Cartan curve in the space form $(M(C), \wp)$ and $\Im$ be a vector field along the curve $\beta$. Then the variation of $\beta$ in the direction of $\Im$ defined by the map $\Gamma: I \times(-\varepsilon, \varepsilon) \rightarrow M(C)$ satisfies $\Gamma(s, 0)=\beta(s), \Im(s)=\frac{\partial \Gamma(s, k)}{\partial s}$. In these cases, we have the following functions of null Cartan longitudinal curves $\beta_{k}(s)=\Gamma(s, k)$ :

1. Speed function $v(s, k)=\left\|\frac{\partial \Gamma(s, k)}{\partial s}\right\|$,

2. Bishop curvature functions $k_{1}(s, k)$ and $k_{2}(s, k)$.

The variations of the speed and the Bishop curvature functions at $k=0$ are calculated as follows:

(a)

$$
\begin{aligned}
\Im(v)= & \left.\left(\frac{\partial v}{\partial k}(s, k)\right)\right|_{k=0}=-v \rho, \\
\Im\left(k_{1}\right)= & \left.\left(\frac{\partial k_{1}}{\partial k}(s, k)\right)\right|_{k=0}=\wp\left(\Re\left(\Im, t_{1}\right) t_{1}+\nabla_{t_{1}}^{2} \Im-k_{2} \nabla_{t_{1}} \Im, n_{1}\right)+2 \rho k_{1}, \\
\Im\left(k_{2}\right)= & \left.\left(\frac{\partial k_{2}}{\partial k}(s, k)\right)\right|_{k=0}=\wp\left(\left(1-k_{1}\right) \Re\left(\Im, t_{1}\right) t_{1}+\left(k_{1}-1\right) \nabla_{t_{1}}^{2} \Im\right. \\
& \left.+k_{2} \nabla_{t_{1}} \Im-k_{1} k_{2} \nabla_{t_{1}} \Im, n_{2}\right)+\left(1-k_{1}\right) \rho^{\prime}-2 \rho k_{2}-2 \rho k_{1} k_{2},
\end{aligned}
$$

where $\rho=g\left(\nabla_{t_{1}} \Im, t_{1}\right)$ and $\Re$ stands for the curvature tensor of $M$.

Proof. Let $\beta: I \subset \mathbb{R} \rightarrow M$ be a null Cartan curve in $(M(C), \wp)$ with constant sectional curvature $C$ and $\Im$ be a vector field along the curve $\beta$. If we take the covariant derivative $\nabla_{\Im}$ of the speed function $v(s, k)$, then it is calculated as follows;

$$
\begin{aligned}
\Im(v) & =\left.\left(\frac{\partial v}{\partial k}(s, k)\right)\right|_{k=0}=\wp\left(\frac{\partial^{2} \Gamma(s, k)}{\partial k \partial s}, v t\right) \\
& =v \wp\left(\nabla_{t_{1}} \Im, t_{1}\right) \\
& =-v \rho .
\end{aligned}
$$

From the Bishop curvature equations, we get

$$
k_{1}=\wp\left(\nabla_{t_{1}} t_{1}, n_{1}\right) .
$$

If we take the covariant derivative $\nabla_{\Im}$ of the Bishop curvature $k_{1}(s, k)$, we obtain

$$
\Im\left(k_{1}\right)=\left.\left(\frac{\partial k_{1}}{\partial k}(s, k)\right)\right|_{k=0}=\wp\left(\nabla_{\Im} \nabla_{t_{1}} t_{1}, n_{1}\right)+\wp\left(\nabla_{t_{1}} t_{1}, \nabla_{\Im} n_{1}\right) .
$$

On the other hand, we have the following two equations;

$$
\nabla_{\Im} \nabla_{t_{1}} t_{1}=-\Re\left(\Im, t_{1}\right) t_{1}+\nabla_{t_{1}} \nabla_{\Im} t_{1}+\nabla_{\left[\Im, t_{1}\right]} t_{1}
$$

and

$$
\begin{aligned}
{[\Im, W] } & =\nabla_{\Im} W-\nabla_{W} \Im \\
& =\frac{\partial^{2} \Gamma(s, k)}{\partial k \partial s}-\frac{\partial^{2} \Gamma(s, k)}{\partial s \partial k} \\
& =0 .
\end{aligned}
$$

Considering the last formulas and the equation $W=v t_{1}$, we get

$$
\left[\Im, v t_{1}\right]=\Im(v) t_{1}-v \nabla_{t} \Im+v\left[\Im, t_{1}\right]=0 .
$$

This provides

$$
\begin{aligned}
{\left[\Im, t_{1}\right] } & =-\frac{1}{v} \Im(v) t_{1} ; \rho=-\frac{1}{v} \Im(v) \\
& =\rho t_{1}
\end{aligned}
$$


Considering the eq.(3.7) and eq.(3.8) with the equation $\left[\Im, t_{1}\right]=\nabla_{\Im} t_{1}-\nabla_{t_{1}} \Im$, we deduce

$$
\nabla_{t_{1}} \nabla_{\Im} t_{1}=\rho^{\prime} t_{1}+\rho \nabla_{t_{1}} t_{1}+\nabla_{t_{1}}^{2} \Im \text {. }
$$

Using the eqs. (3.4)-(3.9) the expression for $V\left(k_{1}\right)$ becomes

$$
\Im\left(k_{1}\right)=\wp\left(-\Re\left(\Im, t_{1}\right) t_{1}+\nabla_{t_{1}}^{2} \Im-k_{2} \nabla_{t_{1}} \Im, n_{1}\right)+2 \rho k_{1} .
$$

Now, we will calculate the variation of the second Bishop curvature

$$
\begin{aligned}
& k_{2}=-\wp\left(\nabla_{t_{1}} t_{1}, n_{2}\right) . \\
& \Im\left(k_{2}\right)=\left.\left(\frac{\partial k_{2}}{\partial k}(s, k)\right)\right|_{k=0}=-\wp\left(\nabla_{\Im} \nabla_{t_{1}} t_{1}, n_{2}\right)-\wp\left(\nabla_{t_{1}} t_{1}, \nabla_{\Im} n_{2}\right) \\
&=-\wp\left(-\Re\left(\Im, t_{1}\right) t_{1}+\rho^{\prime} t_{1}+2 \rho \nabla_{t_{1}} t_{1}+\nabla_{t_{1}}^{2} \Im, n_{2}\right) \\
&-\wp\left(k_{2} t_{1}+k_{1} n_{1}, \nabla_{\Im} n_{2}\right) \\
&=-\wp\left(-\Re\left(\Im, t_{1}\right) t_{1}+\rho^{\prime} t_{1}+\rho \nabla_{t_{1}} t_{1}+\nabla_{t_{1}}^{2} \Im+p \nabla_{t_{1}} t_{1}, n_{2}\right) \\
&+k_{2} \wp\left(\nabla_{\Im} t_{1}, n_{2}\right)+k_{1} \wp\left(\nabla_{\Im} n_{1}, n_{2}\right) .
\end{aligned}
$$

From the Bishop frame equation, we have

$$
n_{1}=-k_{2} t_{1}+n .
$$

Then, the covariant derivative of the eq.(3.13) is computed as

$$
\begin{aligned}
\nabla_{\Im} n_{1} & =-k_{2}^{\prime} \frac{\partial k_{2}}{\partial k} t_{1}-k_{2} \nabla_{\Im} t_{1}+\nabla_{\Im} n ; \quad \frac{\partial k_{2}}{\partial k}=0 \\
& =-k_{2} \nabla_{\Im} t_{1}+\nabla_{\Im} \nabla_{t_{1}} t_{1} \\
& =-k_{2} \rho t_{1}-k_{2} \nabla_{t_{1}} \Im-\Re\left(\Im, t_{1}\right) t_{1}+\rho^{\prime} t_{1}+2 \rho \nabla_{t_{1}} t_{1}+\nabla_{t_{1}}^{2} \Im .
\end{aligned}
$$

If we consider the eq.(3.13) and eq.(3.14), we find

$$
\begin{aligned}
\Im\left(k_{2}\right)= & \left.\left(\frac{\partial k_{2}}{\partial k}(s, k)\right)\right|_{t=0}=\wp\left(\left(1-k_{1}\right) \Re\left(\Im, t_{1}\right) t_{1}+\left(k_{1}-1\right) \nabla_{t_{1}}^{2} \Im\right. \\
& \left.+k_{2} \nabla_{t_{1}} \Im-k_{1} k_{2} \nabla_{t_{1}} \Im, n_{2}\right)+\left(1-k_{1}\right) \rho^{\prime}-2 \rho k_{2}-2 \rho k_{1} k_{2} .
\end{aligned}
$$

Thus, the proof is completed.

Proposition 3.2. Let $\Im(s)$ be a restriction to $\beta(s)$ of a Killing vector field $\Im$ of $M$ then the variations of the Bishop curvature functions and speed function of $\beta$ satisfy:

$$
\Im(v)=\Im\left(k_{1}\right)=\Im\left(k_{2}\right)=0 .
$$

Proof. The local flow $\left\{\phi_{k}\right\}$ created by the Killing vector field $\Im$ consists of local isometries of $M$. Since the variations $\Im(v), \Im\left(k_{1}\right)$ and $\Im\left(k_{2}\right)$ do not depend on the variation $\Gamma$ but only on $\Im(s)$, we can variate $\beta(s)$ in the direction of the vector field $\Im(s)$ as follows:

$$
\beta_{k}(s)=\Gamma(s, k)=: \phi_{k}(\beta(s)) .
$$

The local flow $\phi_{t}$ provides that the velocity function $v(s, k)$ and curvature functions $k_{1}(s, k)$ and $k_{2}(s, k)$ do not depend on the parameter $k$ and thus, we obtain $\Im(v)=\Im\left(k_{1}\right)=$ $\Im\left(k_{2}\right)=0$. Since $M$ is a real-space form with the constant sectional curvature $C$, then the converse of the Proposition 3.2 is stated.

The equations

$$
\Im(v)=\Im\left(k_{1}\right)=\Im\left(k_{2}\right)=0,
$$

consist of a linear system in $\Im(s)$ and the solution space of this system is six dimensional. According to Proposition 3.2, the restriction to $\beta(s)$ of any Killing field of the space form $M(C)$ presents a solution of such a linear system. Since the $M(C)$ is simply connected, its isometry group is also six dimensional. Therefore the space of solutions is the restriction of Killing vector fields of $M(C)$. 
Corollary 3.3. $\Im$ is a Killing vector field along the null Cartan curve $\beta$ if and only if it fulfills the following equations:

i. $\wp\left(\nabla_{t_{1}} \Im, t_{1}\right)=0$,

ii. $\wp\left(\nabla_{t_{1}}^{2} \Im, n_{1}\right)-k_{2} \wp\left(\nabla_{t_{1}} \Im, n_{1}\right)=0$.

Proof. It is clear from Lemma 3.1.

\section{An application: Magnetic curves}

In three-dimensional spaces, a divergence-free vector field generates a magnetic field. If $\Im \in \chi(M)$ is a Killing vector field then it satisfies $\ell_{\Im} \wp=0$. This implies that the Killing vector field on $(M, \wp)$ is divergence free. Thus, for $n=3$, a Killing vector field defines a magnetic field (see [1]).

The Lorentz force related to the magnetic field $F$ is a skew-symmetric operator defined by

$$
\wp(\phi(\omega), \Im)=F(\omega, \varpi),
$$

for all $\omega, \varpi \in \chi(M)$.

The cross product of the vectors $\omega, \varpi \in \chi(M)$ is defined as

$$
\omega \times \varpi=\left(\omega_{2} \varpi_{3}-\omega_{3} \varpi_{2}, \omega_{3} \varpi_{1}-\omega_{1} \varpi_{3}, \omega_{2} \varpi_{1}-\omega_{1} \varpi_{2}\right) .
$$

The mixed product of the vectors $\omega, \varpi \in \chi(M)$ is given by

$$
\wp(\omega \times \varpi, \varrho)=d v_{\wp}(\omega, \varpi, \varrho),
$$

where $d v_{\wp}$ denotes the volume element on $M$.

Lorentz force $\phi$ related to the magnetic field $\Im$ is given by

$$
\phi(\omega)=\Im \times \omega
$$

and the magnetic curves satisfy

$$
\phi(t)=\Im \times t=\nabla_{t} t,
$$

where $\nabla$ is the Levi-Civita connection of $M$.

Proposition 4.1. Let $\left\{t_{1}, n_{1}, n_{2}\right\}$ be Bishop frame and $\left\{k_{1}, k_{2}\right\}$ be Bishop curvatures of null Cartan curve $\beta$. Then, the Lorentz force equations according to the Bishop frame of null Cartan curve $\beta$ is calculated as follows:

$$
\left[\begin{array}{l}
\phi\left(t_{1}\right) \\
\phi\left(n_{1}\right) \\
\phi\left(n_{2}\right)
\end{array}\right]=\left[\begin{array}{ccc}
k_{2} & k_{1} & 0 \\
-\sigma & 0 & k_{1} \\
0 & -\sigma & -k_{2}
\end{array}\right]\left[\begin{array}{c}
t_{1} \\
n_{1} \\
n_{2}
\end{array}\right],
$$

where the function $\sigma(s)$ is quasi-slope associated with each magnetic trajectory of the magnetic field $\Im$.

Proof. Let $\beta$ be a null Cartan magnetic curve in $(M, \wp)$ with the Bishop apparatus $\left\{t_{1}, n_{1}, n_{2}, k_{1}, k_{2}\right\}$. Then, the eq.(4.5) implies

$$
\phi\left(t_{1}\right)=\nabla_{t_{1}} t_{1}=k_{2} t_{1}+k_{1} n_{1} .
$$

Since $\phi\left(n_{1}\right) \in \operatorname{span}\left\{t_{1}, n_{1}, n_{2}\right\}$, we can express

$$
\phi\left(n_{1}\right)=\lambda t_{1}+\mu n_{1}+\zeta n_{2} .
$$

Then, we obtain the following results

$$
\begin{aligned}
\lambda & =-\wp\left(\phi\left(n_{1}\right), n_{2}\right)=-\sigma, \\
\mu & =\wp\left(\phi\left(n_{1}\right), n_{1}\right)=0, \\
\zeta & =\wp\left(\phi\left(n_{1}\right), t_{1}\right)=-\wp\left(\phi\left(t_{1}\right), n_{1}\right)=k_{1} .
\end{aligned}
$$


As a consequence the vector field $\phi\left(n_{1}\right)$ is obtained as

$$
\phi\left(n_{1}\right)=-\sigma t_{1}+k_{1} n_{2} .
$$

Finally, a similar computation leads to

$$
\phi\left(n_{1}\right)=-\sigma n_{1}-k_{2} n_{2} .
$$

Proposition 4.2. Let $\beta$ be a null Cartan magnetic curve in $(M(C), \wp)$. Then $\beta$ is a magnetic trajectory of a magnetic field $\Im$ if and only if $\Im$ can be written along $\beta$ as

$$
\Im(s)=\sigma t_{1}-k_{2} n_{1}+n_{2},
$$

where $k_{1}$ and $k_{2}$ are Bishop curvatures of $\beta$ and the function $\sigma(s)$ is quasislope associated with the magnetic curves of the magnetic field $\Im$.

Proof. Let $\beta$ be a null Cartan magnetic curve of a magnetic field $\Im$. Then, we can write

$$
\Im(s)=\eta t+\varsigma n_{1}+\delta n_{2},
$$

where $\eta, \varsigma$ and $\delta$ are certain functions along a trajectory of $\Im$ and assume $\Im$ does not vanish on $\beta$. When the equations (4.4)-(4.6) are used, we calculate $\eta=\sigma, \varsigma=-k_{2}$ and $\delta=k_{1}$. Conversely, if we assume that eq.(4.13) holds, then we get $\Im \times t_{1}=\phi\left(t_{1}\right)$. Thus, $\beta$ is a magnetic curve of the magnetic vector field $\Im$.

Remark 4.3. Under the pseudo-arc parametrization, generalized null cubics are represented as null helices of zero lightlike curvature. Moreover, such curves are unique up to Lorentz transformation. Null helices of zero lightlike curvature are cubic curves with respect to the pseudo-arc. Null helices of zero lightlike curvature are called null cubics [14].

Theorem 4.4. Let $\beta$ be a null Cartan magnetic curve and $\Im$ be a Killing vector field on the space form $(M(C), \wp)$. If $\beta$ is one of the null Cartan magnetic trajectories of $(M(C), \wp ; \Im)$, then $\beta$ is a null Cartan cubic curve with the second Bishop curvature $k_{2}$ vanishes.

Proof. Let $\Im$ be a magnetic field in $(M(C), \wp)$. Then, $\Im$ satisfies eq.(4.12). By differentiating eq.(4.12), we get

$$
\nabla_{t_{1}} \Im=\sigma^{\prime} t_{1}-k_{2}^{\prime} n_{1}+\sigma k_{2} t_{1}+\sigma n_{1}-k_{2} n_{2} .
$$

Using Lemma 3.1, we compute

$$
\begin{aligned}
\Im(v) & =v \wp\left(\nabla_{t_{1}} \Im, t_{1}\right) \\
& =v \wp\left(\sigma^{\prime} t_{1}-k_{2}^{\prime} n_{1}+\sigma k_{2} t_{1}+\sigma n_{1}-k_{2} n_{2}, t_{1}\right) \\
& =-k_{2} .
\end{aligned}
$$

From Proposition 3.2, we obtain $k_{2}(s)=0$. This gives $\beta$ is a null Cartan cubic. Then differentiation of eq.(4.12) provides

$$
\nabla_{t_{1}}^{2} \Im=\sigma^{\prime \prime} t_{1}+2 \sigma^{\prime} n_{1}+\sigma n_{2} .
$$

Also, we have the following equation;

$$
\Re\left(\Im, t_{1}\right) t_{1}=C\left(\wp\left(t_{1}, \Im\right) t_{1}-\wp\left(t_{1}, t_{1}\right) \Im\right) .
$$

Since $\beta$ is a null Cartan curve, then we have $\wp\left(t_{1}, t_{1}\right)=0$ and we calculate $\wp\left(t_{1}, \Im\right)=-1$. We deduce

$$
\Re\left(\Im, t_{1}\right) t_{1}=-C t_{1} .
$$

Considering the eq.(4.16) and eq.(4.18) with second equation in Lemma 3.1, we have the following equation;

$$
\begin{aligned}
\Im\left(k_{1}\right) & =\wp\left(\Re\left(\Im, t_{1}\right) t_{1}+\nabla_{t_{1}}^{2} \Im, n_{1}\right)=0 \\
& =2 \sigma^{\prime}=0,
\end{aligned}
$$


which shows that $\sigma$ is a constant.

Corollary 4.5. Let $\beta$ be a null Cartan magnetic curve in the simply connected space form $(M(C), \wp)$. Then $\beta$ has the following parametric representation:

$$
\beta(s)=c_{1} s^{3}+c_{2} s^{2}+c_{3} s+c_{4},
$$

where $c_{i}, \in \mathbb{R}_{1}^{3}, i=1,2,3,4$.

Proof. It is clear from Theorem 4.4 and Bishop frame equations.

Example 4.6. If we choose $c_{1}=\left(\frac{1}{8}, 0, \frac{1}{8}\right), c_{2}=(0,1,0), c_{3}=\left(\frac{2}{3}, 0,-\frac{2}{3}\right)$, and $c_{4}=(0,0,0)$, then we obtain null Cartan magnetic curve $\beta$ parameterized as follows:

$$
\beta(s)=\left(\frac{s^{3}}{8}+\frac{2 s}{3}, \frac{s^{2}}{2}, \frac{s^{3}}{8}-\frac{2 s}{3}\right) .
$$

The curve has the following Bishop curvatures:

$$
k_{1}(s)=1, k_{2}(s)=0 .
$$

By using Theorem 4.4, we can easily calculate that $\sigma=1$, and so $\beta$ is a null Cartan magnetic curve. Then, the variational vector field is calculated as;

$$
\Im(s)=\left(\frac{3 s^{2}}{4}+\frac{17}{12}, s, \frac{3 s^{2}}{4}+\frac{1}{12}\right) .
$$

The image of the null Cartan magnetic trajectory is plotted in Figure 1.

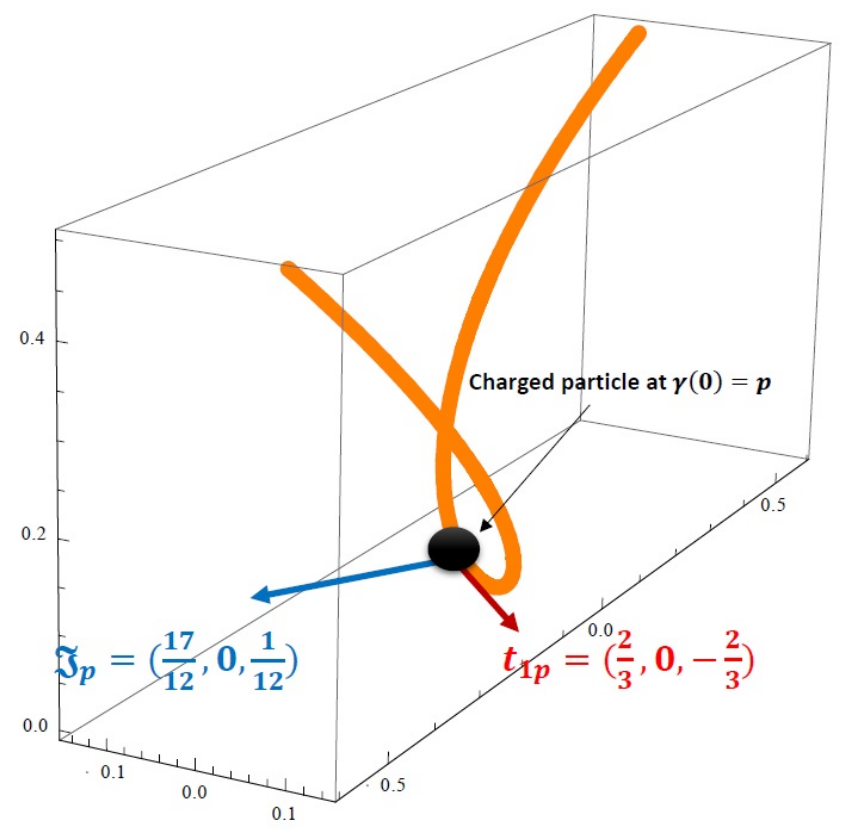

Figure 1. Null Cartan magnetic curve with $\sigma=1$.

\section{Physical meaning of the results}

The behavior of the charged particle has been examined in terms of the Lorentz force $F$. The Lorentz force acting on a particle can be expressed by the electric charged $q$, tangent vector field $t$, electric field $E$ and magnetic vector field $\Im$ as follows;

$$
F=q(E+t \times \Im) .
$$


When electric current flow moves through a conductor $(M, \wp)$ and perpendicular to an applied magnetic field $\Im$, then the Lorentz force has the form as follows;

$$
F=q(t \times \Im) .
$$

In this article, we use the form in eq.(5.1). While a charged particle moves along a null Cartan curve in the Killing magnetic vector field, it traces a helical trajectory. Moreover, the axis of the helix is parallel to the Killing magnetic vector field $\Im$. Furthermore, since the Killing magnetic field has the form in eq.(4.12), the charged particle does not move parallel to the Killing magnetic field $\Im$. Furthermore, the magnetic field can have no effect on an isolated charge. In Figure 2, we illustrate the movement of the charged particle in a Killing magnetic vector field $\Im$.

\section{Conclusions and discussion}

The main purpose of this study is to present null Cartan curve variations and their applications. In this paper, we establish variations of the Bishop frame curvatures along a pseudo null curve in a semi-Riemannian space form. Then, we obtain Killing equations using these variations. Finally, as an application, the charged particle trajectories along the null Cartan curves are determined by using the Killing vector fields. The characterizations of these trajectories are generally given in terms of the variations related to the Frenet frame rather than obtaining the variations of Bishop frame curvatures (see [16]). This makes the given characterizations more complex. We are able to give simple and physically significant characterizations. In conclusion, we reach that the charged particle follows a helical trajectory in its movement along the null Cartan curve in the Killing magnetic vector field.

In comparison with the previous studies, Kazan et al. examined the null Cartan magnetic trajectories and obtained some characterizations by using the Frenet frame equations [15]. However, the authors did not present the curvatures or the parametric representations of these curves. In this paper, Killing equations enabled defining the curvatures and the parametric representations of all null Cartan magnetic trajectories. Moreover, We have presented the physical interpretation of the theoretical results by means of the Killing equations. In $[1,2,4,5,19]$, the authors examined the magnetic trajectories in 3-dimensional Riemannian and semi-Riemannian space forms and reached the following results; when a charged particle moves in a static magnetic field, it follows a helical path. If the velocity vector and the magnetic vector fields are perpendicular to each other, then the particle traces the circular trajectories. In [17], the author examined the charged particle motion along the pseudo null curves and concluded that the charged particle had similar trajectories with the non-null curves. Different results are found compared to the previous studies in this study. It is concluded that the particle follows only helical trajectory along a null Cartan curve in the Killing magnetic field as a different result. The charged particle motion along the null Cartan curve is illustrated in Figure 2.

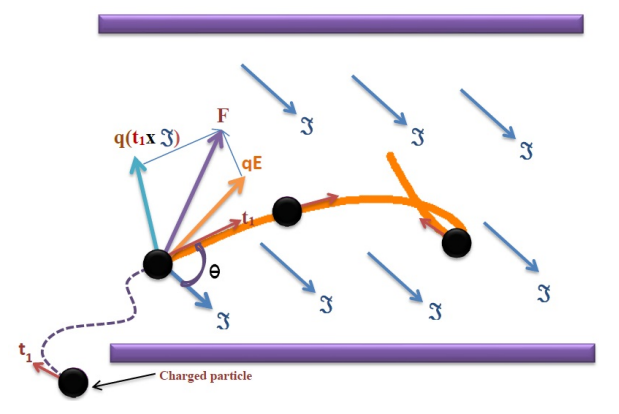

Figure 2. A charged particle moves along the null Cartan helical trajectory. 


\section{References}

[1] M. Barros, J.L. Cabrerizo, M. Fernández, and A. Romero, Magnetic vortex filament flows, J. Math. Phys. 48 (8), 27 pp., 2007.

[2] M. Barros, A. Ferrández, P. Lucas, and M.A. Merono, General helices in the 3dimensional Lorentzian space forms, Rocky Mountain J. Math. 31, 373-388, 2001.

[3] L.R. Bishop, There is more than one way to frame a curve, Amer. Math. Monthly. 82 (3), 246-251, 1975.

[4] Z. Bozkurt, İ. Gök, Y. Yayll, and F.N. Ekmekci, A new approach for magnetic curves in 3D Riemannian manifolds, J. Math. Physics. 55, 053501, 2014.

[5] S.L. Druta-Romaniuc and M.I. Munteanu, Killing magnetic curves in a Minkowski 3-space, Nonlinear Anal. Real World Appl. 14, 383-396, 2013.

[6] K.L. Duggal and D.H. Jin, Null Curves and Hypersurfaces of Semi-Riemannian Manifolds, World Scientific, Singapore, 2007.

[7] A. Ferrández, A. Giménez, and P. Lucas, Geometrical particles models on 3D null curves, Physics Letters B 543 (3-4), 311-317, 2002.

[8] A. Ferrández, A. Giménez, and P. Lucas, Relativistic particles and the geometry of $4 D$ null curves, J. Geom. Phys. 57 (10), 2124-2135, 2007.

[9] A. Giménez, Relativistic particles along null curves in 3D Lorenzian space forms, Internat. J. Bifur. Chaos Appl. Sci. Engrg. 20 (9), 2851-2859, 2010.

[10] M. Grbović and E. Nešović, On the Bishop frames of pseudo null and null Cartan curves in Minkowski 3-space, J. Math. Anal. Appl. 461, 219-233, 2018.

[11] L.P. Hughston and W.T. Shaw, Real classical strings, Proc. Roy. Soc. London Ser. A. 414, 415-422, 1987.

[12] L.P. Hughston and W.T. Shaw, Classical strings in ten dimensions, Proc. Roy. Soc. London Ser. A. 414, 423-431, 1987.

[13] L.P. Hughston and W.T. Shaw, Constraint-free analysis of relativistic strings, Classical Quantum Gravity 5, 69-72, 1988.

[14] J. Inoguchi and S. Lee, Null curves in Minkowski 3-space, Int. Electronic J. Geom. 1 (2), 40-83, 2008.

[15] A. Kazan and H.B. Karadag, Magnetic pseudo null and magnetic null curves in Minkowski 3-space, Int. Math. Forum 12 (3), 119-132, 2017.

[16] A. Kazan and H.B. Karadag, Magnetic Curves According to Bishop Frame and Type-2 Bishop Frame in Euclidean 3-Space, British J. Math. Comp. Sci. 22 (4), 1-18, 2017.

[17] Z. Özdemir, Pseudo Null Curve Variations for Bishop Frame in 3D semi-Riemannian Manifold, Int. J. Geom. Methods Modern Phys. 16 (3), 1950043, 2019.

[18] M. Özdemir and A.A. Ergin, Parallel frame of non-lightlike curves, Missouri J. Math. Sci. 20 (2), 127-137, 2008.

[19] Z. Özdemir, İ. Gök, Y. Yaylı and F.N. Ekmekci, Notes on Magnetic Curves in 3D semi-Riemannian Manifolds, Turk. J. Math. 39, 412-426, 2015.

[20] W. T. Shaw, Twistors and strings (Santa Cruz, CA). 337-363 (1986). Amer. Math. Soc., RI, 1988.

[21] H. Urbantke, On Pinl's representation of null curves in $n$ dimensions, In Relativity Today, Budapest, 34-36, 1987, World Sci. Publ., Teaneck, New York, 1988. 